\title{
ON THE COHOMOLOGY OF STABLE TWO STAGE POSTNIKOV SYSTEMS
}

BY

\author{
JOHN R. HARPER
}

\begin{abstract}
We study the cohomology of certain fibre spaces. The spaces are the total spaces of stable two stage Postnikov systems. We study their cohomology as Hopf algebras over the Steenrod algebra. The first theorem determines the cohomology as a Hopf algebra over the ground field, the algebra structure being known previously. The second theorem relates the action of the Steenrod algebra to the Hopf algebra structure and other available structures. The work is in the direction of explicit computations of these structures but is not quite complete with regard to the action of the Steenrod algebra. The ideas of Massey and Peterson [7], Mem. Amer. Math. Soc. No. 74, are used extensively, and mod 2 cohomology is used throughout.
\end{abstract}

Introduction. Let $F \rightarrow E \rightarrow B$ be a two stage Postinkov system with stable $k$ invariant. Under several special assumptions (listed in $\S 3$ ) we study the mod 2 cohomology of $\Omega E$ over the Steenrod algebra $A$. We shall suppress further mention of the coefficient group, since mod 2 cohomology is used exclusively.

A complete description of $H^{*}(\Omega E)$ as an algebra over $Z_{2}$ is available in [3], [11], [6] and [7]. These papers also contain certain information about the other structures. What has been missing are methods for explicitly calculating coproducts for the Hopf algebra structure and the action of the Steenrod algebra. Theorem 3.2, combined with results of [6] and [7], solves the first of these problems and gives partial information about the second.

We use the work of [6] and [7] as our starting point. Our main contribution is Theorem 3.2. It gives coproducts for a certain subset of $H^{*}(\Omega E)$. The fundamental sequence of the two stage Postnikov system gives one a systematic means of extending the information of Theorem 3.2, to obtain the coproduct for $H^{*}(\Omega E)$. The fundamental sequence also provides a systematic means of using the $\mathrm{H}$ structure to obtain information about the $A$-structure. It would be instructive to have the connection between the $H$-structure and $A$-structure expressed in a functorial manner.

The paper is organized as follows. In $\S 1$ terminology and definitions are given. In $\$ 2$ we study special cases.

The proof of Theorem 3.2 appears in $\S 3$. Essentially the proof is a reduction of general cases to one of the special cases of $\S 2$. In $\S 4$ we use the $H$-structure to gain

Received by the editors November 10, 1969.

AMS 1969 subject classifications. Primary 5550; Secondary 5534.

Key words and phrases. Fibration, fibre space, Postnikov system, Hopf algebra, Steenrod algebra, stable $k$-invariant, Massey-Peterson fundamental sequence.

Copyright (C) 1970, American Mathematical Society 
results about the $A$-structure. $\S 5$ contains examples. The reader may prefer to look at $\$ 5$ prior to $\S 4$.

A recent paper of Milgram [8] contains results for cases included in this work. His method is inference from the $H$-structure to the $A$-structure. This method has a fairly long history, see [1], [5] and [10].

Kristensen [4] has announced a method which determines the $A$-structure of two stage spaces. His methods are different from those used here. Different methods are also available in [9].

It is a pleasure to acknowledge information obtained from Donald Anderson, William Massey, Frank Peterson and Hans Salomonsen. Peterson made several helpful remarks about early versions of this work. Salomonsen aided me in improving the proof of Theorem 3.2. Anderson suggested example D of $\S 5$. Massey made a number of suggestions and comments about earlier drafts of this paper. His remarks were especially helpful in directing the exposition into its present form.

C. K. Cheng has informed me that he has obtained the lemmas of $\S 2$ as part of his study of unstable two stage Postnikov systems.

1. Unstable $A$-modules and fibre spaces. In this section we review some of the ideas and results of [7]. We obtain lemmas and constructions which are used in the proofs of the main theorems. We assume the reader is familiar with [7].

Let $A$ denote the mod 2 Steenrod algebra. Let $M$ be a module over $A$. We assume all modules are graded by the nonnegative integers, are locally finite and defined over $Z_{2} . M$ is called unstable if

$$
\operatorname{Sq}^{n} x=0, \quad n>\operatorname{grade} x .
$$

An algebra over $A$ is unstable if it is unstable as a module and

$$
\operatorname{Sq}^{n} x=x^{2}, \quad n=\text { grade } x .
$$

Let $\mathscr{U}(M)$ denote the free unstable $A$-algebra on $M$ as defined in [12]. We let $F_{n}$ denote the free unstable $A$-module on a single generator of grade $n . F_{n}$ is a cyclic $A$-module and can be expressed $\left(F_{n}\right)_{i}=(A \mid B(n))_{i-n}$ where $B(n) \subset A$ is the left ideal of cohomology operations which annihilate all $n$-dimensional classes. It is useful to recall from [12] that a $Z_{2}$-basis for $F_{n}$ consists of all Sq $I$ where $I$ is admissible with excess $\leqq n$. If $K(\pi, n)$ is an Eilenberg-Mac Lane space, then $H^{*}(\pi, n)=\mathscr{U}(X)$ for some unstable $A$-module $X$. See [7, p. 36] for a detailed description of the cases that interest us.

Massey and Peterson introduce the idea of a $\lambda$-module, which is useful in our work.

Definition. Let $X$ be an unstable $A$-module, $X=\left\{X_{n}\right\}$. Define $\lambda: X \rightarrow X$ by $\lambda \mid X_{n}=\mathrm{Sq}^{n}$.

We refer to [7, p. 33] for the theory of $\lambda$-modules. In particular we use the fact $[7$, p. 33] that any locally finite $\lambda$-module $X$ is a direct sum of cyclic $\lambda$-modules. This allows us to obtain a useful decomposition of the vector space $X$. 
Let $X$ be a locally finite unstable $A$-module. Express

$$
X=\sum_{i} Y_{i}, \quad Y_{i} \text { cyclic on } y_{i} \text {. }
$$

Then a $Z_{2}$-basis for $X$ consists of all elements

$$
\lambda^{j} y_{i}, \quad l<\operatorname{order} y_{i} .
$$

We define a collection of subbases, $\left\{C_{k}\right\}_{k \geqq 0}$ by

$$
C_{0}=\left\{y_{i}\right\}, \quad C_{k}=\lambda C_{k-1}, \quad k \geqq 1 .
$$

We let $T_{k}$ be the vector space spanned by $C_{k}$ and note the vector space isomorphism

$$
T_{k} \cong \lambda^{k} X / \lambda^{k+1} X, \quad \lambda^{0}=1 .
$$

When $X$ is a free unstable $A$-module, this decomposition can be given in terms of admissible monomials. We do this for $F_{n}$, the extension to direct sums being obvious.

$$
\begin{aligned}
& C_{0}=\{\operatorname{Sq} I u \mid \operatorname{ex} I<n\}, \\
& C_{k}=\left\{\operatorname{Sq} I u \mid I=\left(i, I^{\prime}\right), \operatorname{Sq} I^{\prime} u \in C_{k-1}, \operatorname{ex} I=n\right\} .
\end{aligned}
$$

In this notation, $u$ denotes a generator of grade $n$ of $F_{n}$ and $I$ is admissible.

We next recall the definition in [7] of the functor $\Omega$.

Definition. Let $X$ be an unstable $A$-module. Define $\Omega X$ by $\Omega X=\left\{(\Omega X)_{n}\right\}$ where $(\Omega X)_{n}=(X / \lambda X)_{n+1}$.

Recall that the image and kernel of $\lambda$ are $A$-submodules, thus $\Omega X$ is an $A$ module. It is easily checked to be unstable. A map $\sigma: X \rightarrow \Omega X$ of degree -1 is the projection.

If $W$ is a simply connected generalized Eilenberg-Mac Lane space with $H^{*}(W)=\mathscr{U}(X)$, then $H^{*}(\Omega W)=\mathscr{U}(\Omega X)$ and the suspension in the path-loop fibration $\Omega W \rightarrow P W \rightarrow W$ is just $\sigma: X \rightarrow \Omega X$.

We conclude this section with a definition of a map which is useful for describing coproducts. It is not intended as a structure preserving map. $\otimes$ means $\otimes_{z_{2}}$ throughout this paper.

Definition. Let $X$ be an unstable $A$-module and $\left\{C_{k}\right\}$ be a $Z_{2}$-basis as described above. Define $D: X \rightarrow X \otimes X$ by $D(x)=x \otimes x$ on basis elements; $D\left(\sum x\right)=\sum D(x)$ on sums of basis elements.

2. Coproducts in certain $H$-spaces. Let $B_{1}$ and $B_{2}$ be generalized EilenbergMac Lane spaces such that $B_{1}$ has the weak homotopy type of $\Omega B_{2}$. We write $H^{*}\left(B_{2}\right)=\mathscr{U}(X)$. Let $G_{2}$ be the principal fibre space over $B_{2}$ induced by a map $h: B_{2} \rightarrow K_{n}$. Here as elsewhere $K_{n}$ denotes $K\left(Z_{2}, n\right)$. Let $\theta_{n}$ be the fundamental class of $K_{n}$ and assume $h^{*}\left(\theta_{n}\right) \in X$. Let $G_{1}=\Omega G_{2}$. If $G_{1}$ has the weak homotopy type of $B_{1} \times K_{n-2}$ then it follows that $h^{*}\left(\theta_{n}\right) \in \lambda X$. Of course if $h^{*}\left(\theta_{n}\right)=0$, then this holds with respect to the $H$-structure on $G_{1}$ via loop multiplication. However if 
$h^{*}\left(\theta_{n}\right) \neq 0$, then $G_{1}$ need not have the $H$-structure displayed by the splitting when considered with its loop multiplication. See [1] and [10] for examples.

The following lemmas calculate coproducts on a certain class $\eta_{n-2} \in H^{n-2}\left(G_{1}\right)$. This class restricts to the fundamental class of the fibre. The results are used in $\S 3$.

We use the vector space decomposition of $X$ described in $\S 1$,

$$
X=\sum_{k \geqq 0} T_{k} .
$$

By hypotheses, $h^{*}\left(\theta_{n}\right) \in \sum_{k \geqq 1} T_{k}$. We distinguish three possibilities,

Case 1. $h^{*}\left(\theta_{n}\right) \in T_{1}$;

Case 2. $h^{*}\left(\theta_{n}\right)$ has nonzero components in both $T_{1}$ and $\sum_{k \geq 2} T_{k}$;

Case 3. $h^{*}\left(\theta_{n}\right) \in \sum_{k \geq 2} T_{k}$.

Let $\sigma$ be the suspension in $B_{1} \rightarrow P B_{2} \rightarrow B_{2}$. Note that under the hypotheses on $h^{*}\left(\theta_{n}\right)$ both $p^{*}: H^{*}\left(B_{2}\right) \rightarrow H^{*}\left(G_{2}\right)$ and $(\Omega p)^{*}: H^{*}\left(B_{1}\right) \rightarrow H^{*}\left(G_{1}\right)$ are monic on $T_{0}$ and $\Omega T_{0}$ respectively. Let $q=\Omega p$.

LEMMA 2.1. In Case 1 we can write $h^{*}\left(\theta_{n}\right)=\sum_{i} \lambda x_{i}$ with each $x_{i} \in C_{0}$. The coproduct $\Delta$ on $\eta_{n-2}$ is

$$
\Delta\left(\eta_{n-2}\right)=1 \otimes \eta_{n-2}+D q^{*}\left(\sum_{i} \sigma x_{i}\right)+\eta_{n-2} \otimes 1 .
$$

Proof. Let $x=\sum_{i} x_{i}$. Look at the Serre spectral sequence for $G_{1} \rightarrow P G_{1} \rightarrow G_{2}$. The transgression $\tau$ satisfies $\tau\left(q^{*} \sigma x\right)=p^{*} x$. Since $p^{*}(\lambda x)=\left(p^{*} x\right)^{2}=0, Z=\sum_{i} q^{*} \sigma\left(x_{i}\right)$ $\otimes p^{*} x_{i}$ must be killed by an element which for dimensional reasons comes from the fibre. The only possiblity is an element in $H^{*}\left(G_{1}\right)$ of the form $\eta_{n-2}+\sum q^{*} \beta_{j}$, where $\beta_{j}$ are decomposibles under the cup product from $H^{*}\left(B_{1}\right)$. By making an appropriate change of basis in the cohomology algebra $H^{*}\left(G_{1}\right)$, we can take $\eta_{n-2}$ as the class killing $Z$. The coproduct formula now follows from the fact that the Serre spectral sequence is a spectral sequence of Hopf algebras.

In the next two lemmas we drop the $q^{*}$ and $p^{*}$ to simplify the notation. We place complicated superscripts and subscripts in [ ]. A [ ] following $d$ is a subscript, otherwise a superscript.

LEMMA 2.2. In Case 2 we can write $h^{*}\left(\theta_{n}\right)=\sum_{i \in I} \lambda x_{i}+\sum_{j \in J} \lambda\left[k_{j}\right] y_{j}$ where $k_{j} \geqq 2$, and $\left\{x_{i}\right\},\left\{y_{j}\right\}$ are contained in $C_{0}$. The coproduct on $\eta_{n-2}$ is

$$
\Delta\left(\eta_{n-2}\right)=1 \otimes \eta_{n-2}+D\left(\sum_{i \in I} \sigma x_{i}\right)+\eta_{n-2} \otimes 1 .
$$

Proof. We look at the same spectral sequence as before. In $H^{*}\left(G_{2}\right)$ we have $\sum_{I} \lambda x_{i}=\sum_{J} \lambda\left[k_{j}\right] y_{j} \neq 0$. Let $r$ and $\left\{r_{j}\right\}$ be integers such that

$$
d_{r}\left(\sigma x_{i}\right)=x_{i} \text { and } d\left[r_{j}\right]\left(\sigma y_{j}\right)=y_{j} .
$$

Since $k_{j} \geqq 2$ we have $r_{j}<r$ for all $j$. Since

$$
d\left[r_{j}\right]\left(\sigma y_{j} \otimes y_{j}[m-1]\right)=y_{j}[m]=\lambda\left[k_{j}\right] y_{j},
$$


where $m=2\left[k_{j}\right]$, we have

$$
\left\{\sum_{J} \lambda\left[k_{j}\right] y_{j}\right\}=0
$$

in $E_{r}$ of the spectral sequence. Now

$$
d_{r}\left\{\sum_{I} \sigma x_{i} \otimes x_{i}\right\}=\left\{\sum_{I} \lambda x_{i}\right\}=\left\{\sum_{J} \lambda\left[k_{j}\right] y_{j}\right\}=0 .
$$

Hence this class must be killed and as before the dimensions involved imply it must be killed from the fibre. The argument is completed as in Lemma 2.1.

Lemma 2.3. In Case 3 we can write $h^{*}\left(\theta_{n}\right)=\sum_{J} \lambda\left[k_{j}\right] y_{j}$ with $k_{j} \geqq 2$ and $y_{j} \in C_{0}$. Then $\eta_{n-2}$ is primitive.

Proof. We again look at the sequence for $G_{1} \rightarrow P G_{1} \rightarrow G_{2}$. Let $\left\{r_{j}\right\}$ be integers such that

Let $r=\max _{J}\left\{r_{j}\right\}$. Then

$$
d\left[r_{j}\right]\left(\sigma y_{j}\right)=y_{j}
$$

$$
\begin{aligned}
d_{r}\left\{\sum_{J} \sigma y_{j} \otimes y_{j}[m-1]\right\} & =\left\{\sum_{J} y_{j}[m-1]\right\} \\
& =\left\{\sum_{J} \lambda\left[k_{j}\right] y_{j}\right\}=0 \text { in } E_{r}, \text { where } m=2\left[k_{j}\right] .
\end{aligned}
$$

Let $Z=\left\{\sum_{\mathrm{J}} \sigma y_{j} \otimes y_{j}[m-1]\right\}$ in $E_{\mathrm{r}}$. Since $Z$ cannot survive through the spectral sequence, it must eventually be killed. It is not obvious that $Z$ is hit from the fibre. However, that is still the case. Consider the commutative diagram of fibre spaces

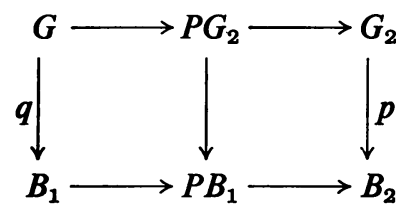

The naturality of the Serre sequence allows us to determine differentials on those elements in the sequence for $(G)$ which come from the sequence for $(B)$. The lowest dimension in which $H^{*}\left(G_{1}\right)$ has an element not in the image of $q^{*}$ is $n-2$. The first such dimension in $H^{*}\left(G_{2}\right)$ is $\geqq n$. Furthermore, for $s \geqq r,\{Z\}$ in $E_{s}(G)$ does not come from $E_{s}(B)$. Thus, $\{Z\}$ must be killed in the sequence for $(G)$ by something which does not come from the sequence for $(B)$. The only possibility is $\left\{\eta_{n-2}\right\}$ (making the same modifications in the cohomology algebra as in Lemma 2.1 if necessary). Let $t$ be the integer such that

$$
d_{t}\left\{\eta_{n-2}\right\}=\{Z\}
$$

The primitivity of $\eta_{n-2}$ will be a consequence of the size of $t$. Since $h^{*}\left(\theta_{n}\right)=\lambda x$ we have that $n$ is even. Since $k_{j} \geqq 2$, we have $t>\frac{1}{2}(n-2)+1$. Suppose $\Delta\left(\eta_{n-2}\right)$ has middle terms $\sum \beta^{\prime} \otimes \beta^{\prime \prime}$. In each summand, one of $\beta^{\prime}$ or $\beta^{\prime \prime}$ has dimension $<\frac{1}{2}(n-2)$. Hence there is an integer $u \leqq \frac{1}{2}(n-1)+1$ such that either $d_{u}$ on $\left\{\beta^{\prime}\right\}$ or 
$\left\{\beta^{\prime \prime}\right\}$ is nonzero. Thus none of the terms $\left\{\beta^{\prime} \otimes \beta^{\prime \prime}\right\}$ survive to $E_{t}$ in the spectral sequence for

$$
G_{1} \times G_{1} \rightarrow P G_{2} \times P G_{2} \rightarrow G_{2} \times G_{2} \quad(G \times G) .
$$

Thus the commutativity of

$$
\begin{gathered}
E_{t}(G) \stackrel{i *}{\longrightarrow} H^{*}\left(G_{1}\right) \\
E_{t}(G \times G) \stackrel{(i \times i)^{*}}{\longrightarrow} \\
H^{*}\left(G_{1} \times G_{1}\right)
\end{gathered}
$$

implies that $\left\{\eta_{n-2}\right\}$ is primitive.

3. The main theorem. We first set down our notation. Let $\xi=\left(E, p, B, F, B_{0}, i, F\right)$ denote a stable two stage Postnikov system; $p: E \rightarrow B, f: B \rightarrow B_{0}$ is the classifying map, and $i: F \rightarrow E$ is the inclusion of the fibre in the total space. We shall use $\iota, K=p^{*}(\iota), \theta$ and $\eta$ generically as fundamental classes of $B, E, B_{0}$ and $F$ respectively. Subscripts on fundamental classes denote dimension.

We make the following assumptions on $\xi$.

(0) $F$ and $B$ are simply connected.

(1) $F$ and $B$ are finite products of Eilenberg-Mac Lane spaces.

(2) The nonzero homotopy groups of the factors of $\boldsymbol{B}$ are infinite cyclic or cyclic of order $2^{k}, k=1,2, \ldots$.

(3) The nonzero homotopy groups of the factors of $B_{0}$ (and $F=\Omega B_{0}$ ) are $Z_{2}$.

(4) $E$ is the principal fibre space induced by an $H$-map $f: B \rightarrow B_{0}$.

We let $W, X, Y, Z$ and $V$ denote free unstable $A$-modules such that $H^{*}\left(B_{0}\right)$ $=\mathscr{U}(V), H^{*}(B)=\mathscr{U}(W), Y=\Omega V, Z=\Omega W$ and $X=\Omega Y$.

Our main theorem is concerned with the coalgebra structure of $H^{*}(\Omega E)$ induced by loop multiplication. Let $\Delta: H^{*}(\Omega E) \rightarrow H^{*}(\Omega E) \otimes H^{*}(\Omega E)$ be the coproduct. Let $g=\Omega f: \Omega B \rightarrow \Omega B_{0}$ and $q=\Omega p: \Omega E \rightarrow \Omega B$. Let $V^{\prime}$ and $Y^{\prime}$ denote $\operatorname{ker} f^{*}$ and ker $g^{*}$ respectively. Let $W^{\prime}$ and $Z^{\prime}$ denote coker $f^{*}$ and coker $g^{*}$ respectively. $R$ and $\mathscr{U}\left(Z^{\prime}\right)$ are used interchangeably and are isomorphic to $H^{*}(\Omega B) / \operatorname{ker} q^{*}$ [2]. Let $\sigma_{M}$ be the cohomology suspension associated with the path-loop fibration $\Omega M \rightarrow P M \rightarrow M . M$ will generally be one of $F, E, B$ or $B_{0}$. Let $X^{\prime}$ be the kernel of the transgression in the fibration $\Omega F \rightarrow \Omega E \rightarrow \Omega B$.

Consider the following sequences and diagrams of unstable $A$ modules. The squares are commutative.

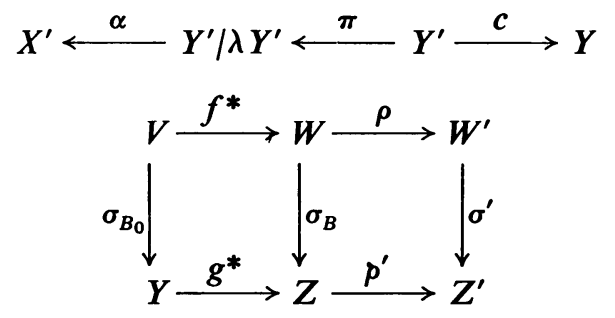


Here $\alpha$ is an isomorphism [7], $\pi, \rho, \rho^{\prime}$ are natural projections, $c$ is inclusion and $\sigma^{\prime}$ is the obvious map induced by $\sigma_{B}$.

We associate with each homogeneous element $x \in X^{\prime}$ an element $z \in Z^{\prime}$ as follows. Let $y \in Y^{\prime}$ such that $\alpha \pi(y)=x$. Let $v \in V$ such that $\sigma_{B_{0}}(v)=c(y)$. Since $\sigma_{B} f^{*}(v)=0$ we have $f^{*}(v)=\lambda w$ for some $w \in W$. Let $z=\sigma^{\prime} \rho(w), z \in Z^{\prime}$.

LEMMA 3.1. $z$ is a uniquely determined element of $Z^{\prime}$.

Proof. $\sigma_{B_{0}}$ is a map of degree -1 and $\lambda$ doubles degrees. Hence $\rho f^{*} \mid \sigma_{B_{0}}^{-1}(\lambda Y)=0$. This and looking at the choices involved in obtaining $z$ give the result.

THEOREM 3.2. For each homogeneous element $x \in X^{\prime}$, there exists an element $e \in H^{*}(\Omega E)$ such that $\Omega i^{*}(e)=x$ and the coproduct is given by

$$
\Delta(e)=1 \otimes e+D q^{*}(z)+e \otimes 1 .
$$

Proof. Let $n=$ degree $x$. Define a map $h: B_{0} \rightarrow K_{n+2}$ by $h^{*}\left(\gamma_{n+2}\right)=v$, where $\gamma_{n+2}$ is the fundamental class and $v$ is as above. We note that neither $v$ nor $h$ are uniquely determined. The effects of this will be discussed later. Consider the following commutative diagram:

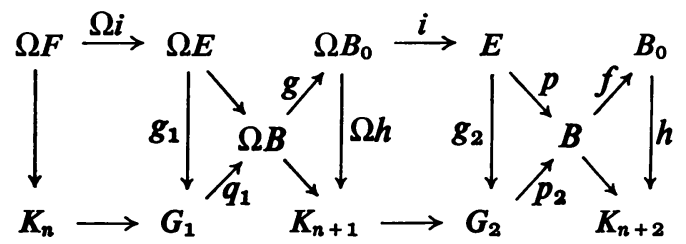

$G_{2}$ is the principal fibre space over $B$ with fibre $K_{n+1}$ and classifying map $h f$. The map $g_{2}$ exists because $(h f) p$ is homotopically trivial. Every space and map to the left of and including $\Omega h$ is obtained by applying $\Omega$ to the corresponding space or map on the right. Hence $g_{1}$ is an $H$-map. Let $\eta_{n} \in H^{n}\left(G_{1}\right)$ be any class which restricts to the fundamental class of $K_{n}$. Then $\Omega i^{*} g_{1}^{*}\left(\eta_{n}\right)=x$. Since $f^{*}(v)=\lambda w$, we have $(\Omega h g)^{*}\left(\gamma_{n+1}\right)=0$. Thus $G_{1} \simeq \Omega B \times K_{n}$ and our lemmas of $\S 2$ apply. Hence we can choose a class $\eta_{n}$ whose coproduct has $D q_{1}^{*}(z)$ as its middle terms. We define $e=q_{1}^{*}\left(\eta_{n}\right)$. Since $q_{1}^{*}$ is a map of Hopf algebras, the theorem follows.

For completeness we discuss the influence of the various choices in the proof. First consider the situation where $v_{1}$ and $v_{2} \in V$ such that $\sigma_{B_{0}}\left(v_{i}\right)=y, i=1,2$. Then $v_{1}=v_{2}+\lambda v_{3}$. Since $f^{*}\left(v_{i}\right)=\lambda w_{i}$ we have $w_{1}=w_{2}+f^{*}\left(v_{3}\right)$. Thus the variation in the middle terms of the coproduct of $\eta_{n}$ given in the argument is

$$
D q_{1}^{*} \sigma_{B} f^{*}\left(v_{3}\right)=D q_{1}^{*} \Omega f^{*} \sigma_{B_{0}}\left(v_{3}\right) .
$$

Hence the coproduct of $e$ is unaffected.

Variation in $v$ is also possible as a result of different choices of $y$ such that $\alpha \pi(y)=x$. Arguments similar to Lemma 3.1 show this choice does not affect the coproduct of $e$. 
The $H$-map $g_{1}$ is not uniquely determined by the construction. However one sees that different choices satisfy $\left(g_{1}-g_{1}^{\prime}\right)^{*}\left(\eta_{n}\right)=$ primitive from $H^{*}(\Omega B)$. Thus the corresponding $e$ 's differ by primitives leaving the coproduct formula unchanged. The possibility of adding primitives to $e$ will be used in $\$ 4$.

Our next remark concerns the relation of Theorem 3.2 to the known splitting $H^{*}(\Omega E) \cong R \otimes \mathscr{U}\left(X^{\prime}\right)$ as algebras over $Z_{2}$. This isomorphism is not natural. Let one be denoted by $\varphi: R \otimes \mathscr{U}\left(X^{\prime}\right) \rightarrow H^{*}(\Omega E)$. In general the coproducts of $\varphi(x)$ and $e$ given in Theorem 3.2 differ by terms of the form $\varphi(\alpha) \otimes \varphi(\beta)+\varphi(\beta) \otimes \varphi(\alpha)$; $\alpha \neq \beta ; \alpha, \beta \in R$. This ambiguity is similar to that encountered in the spectral sequence arguments of $\S 2$.

Finally we indicate one way to use Theorem 3.2 to describe the coalgebra structure of $H^{*}(\Omega E)$. Let $\left\{x_{i}\right\}$ be a homogeneous $Z_{2}$-basis for $X^{\prime}$ and $\left\{e_{i}\right\}$ be elements of $H^{*}(\Omega E)$ satisfying Theorem 3.2. Then by [6], $\{1\} \cup\left\{e_{i}\right\}$ form a simple system of generators for $H^{*}(\Omega E)$ as an algebra over $R$. Hence Theorem 3.2 is enough to calculate coproducts for any element in $H^{*}(\Omega E)$.

4. The action of the Steenrod algebra. Massey and Peterson have reduced the problem of determining the action of $A$ in $H^{*}(\Omega E)$ to the determination of the $A$-module extension represented by the fundamental sequence of $\Omega \xi$,

$$
0 \longrightarrow R \stackrel{q^{*}}{\longrightarrow} P(\Omega \xi) \stackrel{\Omega i^{*}}{\longrightarrow} X^{\prime} \longrightarrow 0 .
$$

Here we treat the problem of calculating the action of $A$ in $P(\Omega \xi)$ as follows. First note that the class $e \in H^{*}(\Omega E)$ of Theorem 3.2 actually lies in $P(\Omega \xi)$. Let $\left\{x_{i}\right\}$ be a homogeneous basis (over $Z_{2}$ ) for $X^{\prime}$. Let $\left\{e_{i}\right\} \subset P(\Omega \xi)$ be such that $\Omega i^{*}\left(e_{i}\right)=x_{i}$ and coproducts on $\left\{e_{i}\right\}$ are given by Theorem 3.2. Let $\theta \in A$ and consider $\Omega i^{*}\left(\theta e_{i}\right)=\sum x_{j}$. Then $\theta e_{i}+\sum e_{j}=q^{*}(r)$ for a unique $r \in R$. The problem is to calculate $r$. Let $e^{\prime}=\theta e_{i}+\sum e_{j}$. We use the following diagram of [7, p. 63], $\bar{\Delta}$ 's denote coproducts with terms of the form $1 \otimes x+x \otimes 1$ subtracted. Only positive gradings are considered.

(5)

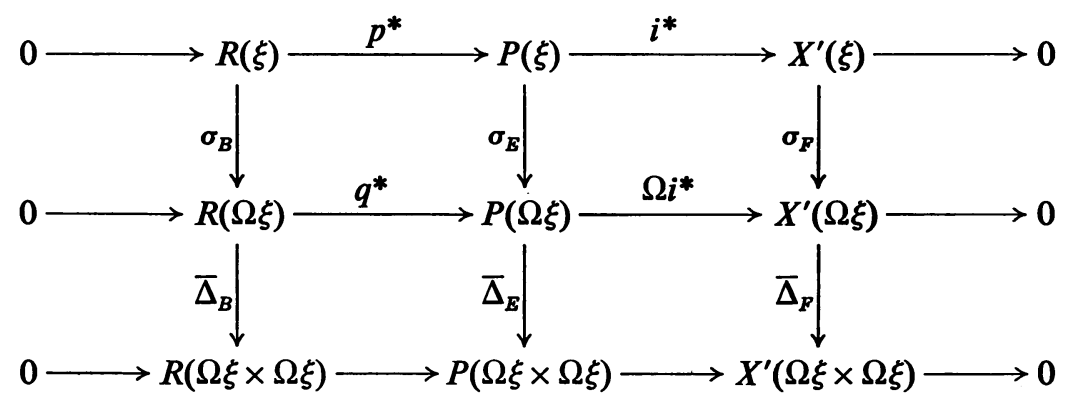

This diagram expresses the naturality properties of fundamental sequences useful in this section. 
One can obtain information about $r$ by studying $\bar{\Delta}_{E}\left(e^{\prime}\right)$ and $\sigma_{\Omega E}\left(e^{\prime}\right)$. The idea is to determine the action of $A$ through an inductive sequence of deloopings, beginning with an easy low dimensional case. But we caution the reader that our results are not complete. Theorems 4.2 and 4.3 indicate the delooping process. In order to use diagram (5) for these theorems, we shift our emphasis from the system $\Omega \xi$ to the system $\xi$. Thus we assume we have Theorem 3.2 for the system $\xi$ and that we are calculating the action of $A$ in $H^{*}(E)$. This shift of emphasis involves nothing new. The assumption (3) on $\xi$ implies there exists a system $\eta$ satisfying (0)-(4) such that $\xi=\Omega \eta,[7$, p. 38].

Let $S \subset R(\Omega \xi)$ denote the $A$-submodule of primitives and consider $\bar{\Delta}_{B}: R(\Omega \xi) / S$ $\rightarrow R(\Omega \xi) \otimes R(\Omega \xi)$ as an $A$-map.

THEOREM 4.1. A unique element $[r] \in R(\Omega \xi) / S$ is determined by the formula $\left(q^{*} \otimes q^{*}\right) \bar{\Delta}_{B}([r])=\bar{\Delta}_{E}\left(e^{\prime}\right)$.

Proof. $H^{*}(\Omega B)$ and hence $R(\Omega \xi)$ are primitively generated as Hopf algebras over $Z_{2}$. This implies $\bar{\Delta}_{B}$ is a monomorphism. The theorem follows by chasing around the lower two lines of (5).

THEOREM 4.2. Let $R(\xi)$ be an exterior algebra over $Z_{2}$. Let $r+S$ denote the coset of $R(\xi)$ determined by Theorem 4.1. A unique element $s \in r+S$ is determined by the formula $q^{*} \sigma_{B}(s)=\sigma_{E}\left(e^{\prime}\right)$. With no assumptions on the type of algebra of $R(\xi)$, the same formula determines a unique element $[s] \in r+S /\left(\operatorname{ker} \sigma_{B} \cap S\right)$.

Proof. If $R(\xi)$ is an exterior algebra then $\sigma_{B}$ is a monomorphism and the first statement follows by a diagram chase in (5). The second statement is clear.

Theorem 4.2 is useful for studying $H^{*}(E)$ if $H^{*}(\Omega E)$ is known as a Hopf algebra over $A$ and one can calculate $\sigma_{E}$. We now show how $\sigma_{E}$ can be calculated. Choose a $Z_{2}$-basis for $X^{\prime}(\xi)$ such that $\sigma_{F}$ maps this basis onto a subbasis of $X^{\prime}(\Omega \xi)$. Let $\left\{f_{k}^{\prime}\right\} \subset P(\xi)$ and $\left\{e_{i}\right\} \subset P(\Omega \xi)$ correspond to these bases as in the beginning of this section.

THEOREM 4.3. There exists a set $\left\{f_{k}\right\} \subset P(\xi)$ such that $i^{*}\left(f_{k}\right)=i^{*}\left(f_{k}^{\prime}\right)$ and the coproducts of $\left\{f_{k}\right\}$ are given by Theorem 3.2 and further:

$$
\sigma_{F}\left(f_{k}\right)=e_{i} \text { if } \sigma_{F} i^{*}\left(f_{k}\right)=\Omega i *\left(e_{i}\right)
$$

and

$$
\sigma_{F}\left(f_{k}\right)=0 \text { if } \sigma_{F} i *\left(f_{k}\right)=0 .
$$

Proof. Note that if $\Omega i^{*}\left(e_{i}\right) \in \operatorname{Im} \sigma_{F}$ then $e_{i}$ is primitive. Hence there is a primitive element $s \in R(\xi)$ such that $q^{*} \sigma_{B}(s)=e_{i}-\sigma_{E}\left(f_{k}^{\prime}\right)$ (in case $\left.\sigma_{E}\left(f_{k}^{\prime}\right)=e_{i}\right)$. Set $f_{k}=f_{k}^{\prime}+p^{*}(s)$. The other case is similar.

The results of this section can be summarized by saying that if $H^{*}(\Omega E)$ is known as a Hopf algebra over $A$, then the action of $A$ can be calculated in $H^{*}(E)$ up to primitives in $\operatorname{ker} \sigma_{B}$. We denote such primitives as type DP (for decomposible and 
primitive). Terms of type DP are the only obstruction to an inductive determination of $H^{*}(E)$ as a Hopf algebra over $A$.

5. Examples. Our examples will illustrate theorems of $\$ 3$ and 4. We work with minimal sets of generators and relations in the sense of [1]. Some simple techniques are used to determine terms of type DP.

We first consider a series of related spaces. Let $E_{n}$ be the principal fibre space over $K\left(Z_{2}, n\right)$ with fibre $K\left(Z_{2}, n+1\right)$ and $k$-invariant $\mathrm{Sq}^{2}$. We keep the same symbols used in describing a system $\xi$.

When $n=1$, Lemma 2.1 applies and we have

Proposition 5.1. $H^{*}\left(E_{1}\right) \cong H^{*}\left(Z_{2}, 1\right) \otimes H^{*}\left(Z_{2}, 2\right)$ with $\Delta\left(\eta_{2}\right)=1 \otimes \eta_{2}+$ $\kappa_{1} \otimes \kappa_{1}+\eta_{2} \otimes 1$ and the splitting holding with respect to the action of $A$.

When $n=2$, we have an example already completely worked out in [7]. Since our methods are somewhat different, we do this case for the sake of comparison. In the following subsection $A$, we have deleted subscripts from many fundamental classes. The understood dimensions are as follows: $\eta$ has dimension 3 , $\iota$ and $\kappa$ have dimension 2.

A. Since $\eta$ transgresses to $\iota^{2}$ we have

$$
\begin{aligned}
\tau(\operatorname{Sq} I \eta) & =(\operatorname{Sq} J \iota)^{2}, & & I=2 J, \\
& =0, & & \text { otherwise. }
\end{aligned}
$$

Here as elsewhere symbols Sq $I$ denote admissible monomials. As in Milgram [8], this implies that $\operatorname{ker} \tau$ is generated as an $A$-module by $\mathrm{Sq}_{1}^{1} \eta$ and $\mathrm{Sq}^{3} \eta$.

The transgression on $\eta$ also shows that

$$
\mathscr{U}\left(Z^{\prime}\right) \cong E(\operatorname{Sq} I, \text { excess } I<2),
$$

where $E$ denotes an exterior algebra. Thus there are no terms of type DP. We write a vector space basis for $\mathscr{U}\left(Z^{\prime}\right)$ in various dimensions:

$\operatorname{dim} 4$ and 6. $\mathscr{U}\left(Z^{\prime}\right)=0$,

$\operatorname{dim} 5 . \kappa \cdot \mathrm{Sq}^{1} \kappa, \mathrm{Sq}^{2} \mathrm{Sq}^{1} \kappa$,

$\operatorname{dim} 7 . \kappa \cdot \mathrm{Sq}^{2} \mathrm{Sq}^{1} \kappa$,

$\operatorname{dim} 8 . \mathrm{Sq}^{1} \kappa \cdot \mathrm{Sq}^{2} \mathrm{Sq}^{1} \kappa$.

Thus we can take $e_{4}=1 \otimes \mathrm{Sq}^{1} \eta$ and $e_{6}=1 \otimes \mathrm{Sq}^{3} \eta$.

Direct computation gives the relations

$$
\mathrm{Sq}^{1} e_{4}=\mathrm{Sq}^{1} e_{6}=\mathrm{Sq}^{2} e_{4}+\mathrm{Sq}^{2} e_{6}=0
$$

in the cohomology of the fibre. In fact one can prove [7] that these are the generating relations.

Since $\mathrm{Sq}^{3} \mathrm{Sq}^{2}=0$ we have $e_{6}$ primitive. Since $\mathrm{Sq}^{1} \mathrm{Sq}^{2}=\mathrm{Sq}^{3}$, Lemma 2.1 gives

$$
\Delta\left(e_{4}\right)=1 \otimes e_{4}+\kappa \otimes \kappa+e_{4} \otimes 1
$$


In $H^{*}\left(E_{2}\right)$ we have the relation

$$
0=\mathrm{Sq}^{1} e_{4}+\lambda\left(\kappa \cdot \mathrm{Sq}^{1} \kappa\right)+\mu\left(\mathrm{Sq}^{2} \mathrm{Sq}^{1} \kappa\right), \quad \lambda, \mu \in Z_{2} .
$$

Theorem 4.1 implies $\lambda=1$. To determine $\mu$ we look at $\sigma: H^{*}\left(E_{2}\right) \rightarrow H^{*}\left(E_{1}\right)$. The restriction to the fibre of $\sigma\left(e_{4}\right)$ is $\mathrm{Sq}^{1} \eta$. Since $\sigma\left(e_{4}\right)$ is primitive, and there are no primitives in dimension 3 , we have

$$
\sigma\left(e_{4}\right)=\mathrm{Sq}^{1} \eta+\kappa \cdot \mathrm{Sq}^{1} \kappa .
$$

Hence $\sigma\left(\mathrm{Sq}^{1} e_{4}\right)=\mathrm{Sq}^{2} \mathrm{Sq}^{1} \kappa$ and $\mu=1$.

Since $\mathrm{Sq}^{1} e_{6}$ and $\mathrm{Sq}^{4} e_{4}$ are primitive $\left(\mathrm{Sq}^{2} \kappa=0\right)$ inspection gives $\mathrm{Sq}^{1} e_{6}=0$ $=\mathrm{Sq}^{4} e_{4}+\mathrm{Sq}^{2} e_{6}$ as the relations in $H^{*}\left(E_{2}\right)$.

Summarizing the above we have

Proposition 5.2. $H^{*}\left(E_{2}\right) \cong E(\operatorname{Sq} I \kappa$, ex $I<2) \otimes \mathscr{U}\left(X^{\prime}\right)$ where $X^{\prime}$ is generated by classes $e_{4}$ and $e_{6}$ restricting to $\mathrm{Sq}^{1} \eta$ and $\mathrm{Sq}^{3} \eta$ respectively. The class $e_{6}$ is primitive while $\Delta\left(e_{4}\right)$ has middle term $\kappa \otimes \kappa$. The basic relations are $\mathrm{Sq}^{1} e_{4}+\kappa \cdot \mathrm{Sq}^{1} \kappa+\mathrm{Sq}^{2} \mathrm{Sq}^{1} \kappa$ $=0, \mathrm{Sq}^{1} e_{6}=0$ and $\mathrm{Sq}^{4} e_{4}+\mathrm{Sq}^{2} e_{6}=0$.

B. We now study $H^{*}\left(E_{3}\right)$. In subsection $\mathrm{B}, \eta$ has dimension 4 and $\kappa$ dimension 3 or 2 , which should be clear in context. An easy argument gives

$$
\operatorname{im} p^{*}=E(\operatorname{Sq} I \kappa, \operatorname{ex} I<2) \text {. }
$$

By considering the transgression on terms of the form $\operatorname{Sq}(I, j) \eta$ with $j=1, \ldots, 4$ and $(I, j)$ admissible, one shows that as an $A$-module, $X^{\prime}$ is generated by $\mathrm{Sq}^{3} \eta$ and $\mathrm{Sq}^{3} \mathrm{Sq}^{1} \eta$. For dimensional reasons we can take $e_{7}=1 \otimes \mathrm{Sq}^{3} \eta$ and $e_{8}=1 \otimes \mathrm{Sq}^{3} \mathrm{Sq}^{1} \eta$. We have $e_{7}$ primitive and the middle term of $\Delta\left(e_{8}\right)$ is $\mathrm{Sq}^{1} \kappa \otimes \mathrm{Sq}^{1} \kappa$ since $\left(\mathrm{Sq}^{3} \mathrm{Sq}^{1}\right) \mathbf{S q}^{2}=\mathrm{Sq}^{5} \mathrm{Sq}^{1}$. Calculating through low dimensions produces generating relations in $X^{\prime}$ as follows (with an abuse of notation):

$$
\mathrm{Sq}^{1} e_{7}=\mathrm{Sq}^{1} e_{8}=\mathrm{Sq}^{3} e_{7}+\mathrm{Sq}^{2} e_{8}=0 .
$$

We do not claim these are a complete set of generating relations.

The basis elements in im $p^{*}$ which concerns us are

$\operatorname{dim} 8.0$,

$\operatorname{dim}$ 9. $\kappa \cdot \mathrm{Sq}^{2} \mathrm{Sq}^{1} \kappa$,

$\operatorname{dim} 10 . \mathrm{Sq}^{1} \kappa \cdot \mathrm{Sq}^{2} \mathrm{Sq}^{1} \kappa, \mathrm{Sq}^{4} \mathrm{Sq}^{2} \mathrm{Sq}^{1} \kappa$.

Thus $\mathrm{Sq}^{1} e_{7}=0$ holds in $H^{*}\left(E_{3}\right)$. Theorem 4.1 applied to the next relation implies $\mathrm{Sq}^{1} e_{8}=0$ holds in $H^{*}\left(E_{3}\right)$. Theorem 4.1 applied to the relation

$$
0=\mathrm{Sq}^{3} e_{7}+\mathrm{Sq}^{2} e_{8}+\lambda\left(\mathrm{Sq}^{1} \kappa \cdot \mathrm{Sq}^{2} \mathrm{Sq}^{1} \kappa\right)+\mu\left(\mathrm{Sq}^{4} \mathrm{Sq}^{2} \mathrm{Sq}^{1} \kappa\right)
$$

implies $\lambda=1$.

We now look at $\sigma: H^{*}\left(E_{3}\right) \rightarrow H^{*}\left(E_{2}\right)$. We have $\sigma\left(e_{7}\right)=e_{6}$ and $\sigma\left(e_{8}\right)=\mathrm{Sq}^{3} e_{4}$. Thus we have

$$
0=\mathrm{Sq}^{3} e_{6}+\mathrm{Sq}^{4} \mathrm{Sq}^{1} e_{4}+\mu\left(\mathrm{Sq}^{4} \mathrm{Sq}^{2} \mathrm{Sq}^{1} \kappa\right)=0
$$


in $H^{*}\left(E_{2}\right)$. But using Proposition 5.2 shows $\mathrm{Sq}^{3} e_{6}=0$ and $\mathrm{Sq}^{4} \mathrm{Sq}^{1} e_{4}+\mathrm{Sq}^{4} \mathrm{Sq}^{2} \mathrm{Sq}^{1} \kappa$ $=0$, hence $\mu=1$. We summarize this as

Proposition 5.3. $H^{*}\left(E_{3}\right) \cong E(\mathrm{Sq} I \kappa$, ex $I<2) \otimes \mathscr{U}\left(X^{\prime}\right)$. $X^{\prime}$ is generated by $e_{7}$ and $e_{8}$ restricting to $\mathrm{Sq}^{3} \eta$ and $\mathrm{Sq}^{3} \mathrm{Sq}^{1} \eta$ respectively. The class $e_{7}$ is primitive and $\Delta\left(e_{8}\right)$ has middle term $\mathrm{Sq}^{1} \kappa \otimes \mathrm{Sq}^{1} \kappa$. In low dimensions the basic relations are $\mathrm{Sq}^{1} e_{7}=0$, $\mathrm{Sq}^{1} e_{8}=0$ and

$$
0=\mathrm{Sq}^{3} e_{7}+\mathrm{Sq}^{2} e_{8}+\mathrm{Sq}^{1} \kappa \cdot \mathrm{Sq}^{2} \mathrm{Sq}^{1} \kappa+\mathrm{Sq}^{4} \mathrm{Sq}^{2} \mathrm{Sq}^{1} \kappa
$$

In the next example we shall mainly be concerned with ascertaining whether or not terms of type DP are in relations. Let $E_{n}$ be the principal fibre space over $K\left(Z_{2}, n\right)$ with fibre $K\left(Z_{2}, n+2\right)$ and $k$-invariant $\mathrm{Sq}^{3}$. By our methods one can show

Proposition 5.4. $H^{*}\left(E_{3}\right) \cong E\left(\mathrm{Sq} I \kappa_{3}\right.$, ex $\left.I<3\right) \otimes \mathscr{U}(N) . N$ is generated by $e_{6}$ and $e_{8}$ restricting to $\mathrm{Sq}^{1} \eta_{5}$ and $\mathrm{Sq}^{3} \eta_{5}$ respectively. The class $e_{6}$ is primitive and $\Delta\left(e_{8}\right)$ has middle term $\mathrm{Sq}^{1} \kappa_{3} \otimes \mathrm{Sq}^{1} \kappa_{3}$. The generating relations are $\mathrm{Sq}^{1} e_{6}=0, \mathrm{Sq}^{1} e_{8}=0$ and $\mathrm{Sq}^{5} e_{6}+\mathrm{Sq}^{3} e_{8}+\mathrm{Sq}^{1} \kappa_{3} \mathrm{Sq}^{3} \mathrm{Sq}^{1} \kappa_{3}=0$.

C. We now study $H^{*}\left(E_{4}\right)$. In subsection $\mathrm{C}, \eta$ without a subscript has dimension 6 and $\kappa$ without a subscript has dimension 4 or 3 as the context implies. We find that $\mathrm{Sq}^{1} \eta$ and $\mathrm{Sq}^{5} \eta$ generate $X^{\prime}$ through dimensions $\leqq 15$. Furthermore, through dimensions $\leqq 12$ the generating relations are $\mathrm{Sq}^{1}\left(\mathrm{Sq}^{1} \eta\right)=0$ and $\mathrm{Sq}^{1}\left(\mathrm{Sq}^{5} \eta\right)=0$. Since $\mathrm{Sq}^{1} \mathrm{Sq}^{3}=0$ we have $e_{7}$ primitive. Since $\mathrm{Sq}^{5} \mathrm{Sq}^{3}=0$ we have $e_{11}$ primitive. Direct calculation in dimensions $\leqq 12$ gives

$$
\operatorname{im} p^{*} \cong P(\operatorname{Sq} I \kappa, \text { ex } I \leqq 2, I=2 J) \otimes E(\operatorname{Sq} I \kappa, \text { ex } I \leqq 2, I \neq 2 J),
$$

where $P$ denotes a polynomial algebra. Thus we have to determine the coefficients in the following:

$$
0=\mathrm{Sq}^{1} e_{7}+\lambda \mathrm{Sq}^{3} \mathrm{Sq}^{1} \kappa+\mu \mathrm{Sq}^{4} \kappa
$$

and

$$
0=\mathrm{Sq}^{1} e_{11}+\nu \mathrm{Sq}^{5} \mathrm{Sq}^{2} \mathrm{Sq}^{1} \kappa+\pi \mathrm{Sq}^{6} \mathrm{Sq}^{2} \kappa .
$$

Let $\sigma: H^{*}\left(E_{4}\right) \rightarrow H^{*}\left(E_{3}\right)$. We have

$$
\sigma\left(e_{7}\right)=e_{6}+\rho \mathrm{Sq}^{2} \mathrm{Sq}^{1} \kappa .
$$

Let $\tilde{e}_{7}=e_{7}+\rho \mathrm{Sq}^{2} \mathrm{Sq}^{1} \kappa$. Then $\sigma\left(\tilde{e}_{7}\right)=e_{6}$. Applying $\sigma$ to the relation

$$
0=\mathrm{Sq}^{1}\left(\tilde{e}_{7}\right)+(\lambda+\rho) \mathrm{Sq}^{3} \mathrm{Sq}^{1} \kappa+\mu \mathrm{Sq}^{4} \kappa
$$

gives

$$
0=\mathrm{Sq}^{1}\left(e_{6}\right)+(\lambda+\rho) \mathrm{Sq}^{3} \mathrm{Sq}^{1} \kappa .
$$

Since $\mathrm{Sq}^{1} e_{6}=0$ we have $\lambda+\rho=0$ in the modified relation.

To determine $\mu$ we observe that for all $n \geqq 4$ we have classes $e_{n+3}$ and $e_{n+7}$ in $H^{*}\left(E_{n}\right)$ restricting to $\mathrm{Sq}^{1} \eta_{n+2}$ and $\mathrm{Sq}^{5} \eta_{n+2}$ in the fibre. We can always make 
modifications of the above sort so that

$$
0=\mathrm{Sq}^{1} e_{n+3}+\mu \mathrm{Sq}^{4} \kappa_{n}
$$

and

$$
\sigma\left(e_{n+3}\right)=e_{n+2} \in H^{*}\left(E_{n-1}\right) .
$$

If we apply $\mathrm{Sq}^{1}$ to this relation, we obtain

$$
0=\mu \mathrm{Sq}^{5} \kappa_{n} \text {. }
$$

But for $n \geqq 5$ we have $\mathrm{Sq}^{5} \kappa_{n}=\mathrm{Sq}^{4} \mathrm{Sq}^{1} \kappa_{n} \neq 0$. Thus for $n \geqq 5, \mu=0$. Suspending $H^{*}\left(E_{4}\right)$ then gives $\mu=0$.

The relation involving $\mathrm{Sq}^{1} e_{11}$ is worked out similarly. We can arrange things so that

$$
\sigma\left(e_{11}\right)=\mathrm{Sq}^{4} e_{6}+\mathrm{Sq}^{2} e_{8}+\mathrm{Sq}^{1} \kappa \cdot \mathrm{Sq}^{2} \mathrm{Sq}^{1} \kappa .
$$

This implies $\nu=0$. Again looking in $H^{*}\left(E_{n}\right)$ for $n$ large, we find that $\mathrm{Sq}^{9} \kappa_{n}$, $\mathrm{Sq}^{8} \mathrm{Sq}^{1} \kappa_{n}, \mathrm{Sq}^{7} \mathrm{Sq}^{2} \kappa_{n}, \mathrm{Sq}^{6} \mathrm{Sq}^{2} \mathrm{Sq}^{1} \kappa_{n}$ span a 3-dimensional subspace of $H^{n+9}\left(E_{n}\right)$. This implies $\pi=0$.

D. Our final example is based on the exact sequence of groups (group operation addition)

$$
0 \longrightarrow Z_{a} \stackrel{i}{\longrightarrow} Z_{b} \stackrel{a}{\longrightarrow} Z_{b} \stackrel{j}{\longrightarrow} Z_{a} \longrightarrow 0
$$

where $a=2^{k}, b=2^{f}$ with $k \leqq f$. Here the middle map is multiplication by $a, i(1)=b / a$ and $j(1)=1$. I am indebted to Don Anderson for suggesting this example. It goes with the example in $[7$, p. 41$]$ in indicating the problems when the fibre fails to satisfy condition (3) of $\$ 1$.

Let $E$ be the principal fibre space over $K\left(Z_{b}, n\right)$ with fibre $K\left(Z_{b}, n-1\right)$ and $k$-invariant

$$
a_{\iota_{n}} \in H^{n}\left(Z_{b}, n ; Z_{b}\right) .
$$

The simplest cases illustrating the point are when $f=2, k=1,2$. If $k=2$ then $E \simeq K\left(Z_{4}, n-1\right) \times K\left(Z_{4}, n\right)$ with this splitting holding as $H$-spaces. If $k=1$ we look at the homotopy sequence for $E$,

$$
0 \longrightarrow \pi_{n}(E) \longrightarrow Z_{4} \stackrel{2}{\longrightarrow} Z_{4} \longrightarrow \pi_{n-1}(E) \longrightarrow 0 .
$$

Hence $\pi_{n}(E)=\pi_{n-1}(E)=Z_{2}$. As an algebra

$$
H^{*}(E) \cong H^{*}\left(Z_{4}, n-1\right) \otimes H^{*}\left(Z_{4}, n\right) .
$$

Let $u_{n-1}, v_{n}, u_{n}, v_{n+1}$ be the generators of the factors. These generators are all primitive and the extension possibilities are represented as

$$
\mathrm{Sq}^{1} u_{n-1}=\lambda u_{n}, \quad \mathrm{Sq}^{1} v_{n}=\mu v_{n+1} .
$$

If $n$ is big enough, the extension problem lies in the stable range where the Adams 
spectral sequence [2] converges to the actual homotopy of $E$. Then $\lambda=\mu=1$ are the only possibilities compatible with the Adams spectral sequence.

With other choices of $f$ and $k$ we can obtain trivial $A$-extensions and nontrivial extensions by higher order Bockstein operators.

Massey has pointed out that a better result can be obtained by looking at the Postnikov system of $E$. The $k$-invariant is either 0 or $\mathrm{Sq}^{2}$, but $\mathrm{Sq}^{2} u_{n-1} \neq 0$ so the $k$-invariant is 0 . Hence $E \simeq K_{n-1} \times K_{n}$.

We have included the spectral sequence argument to indicate a way that the homotopy of $E$ influences the $A$-structure of $H^{*}(E)$. The same type of argument (with a lot of computation) gives the results of subsection $\mathrm{C}$.

\section{BIBLIOGRAPHY}

1. J. F. Adams, On the non-existence of elements of Hopf invariant one, Ann. of Math. (2) 72 (1960), 20-104. MR 25 \#4530.

2. - On the structure and applications of the Steenrod algebra, Comment Math. Helv. 32 (1958), 180-214. MR 20 \#2711.

3. L. Kristensen, On the cohomology of spaces with two non-vanishing homotopy groups, Math. Scand. 12 (1963), 83-105. MR 28 \#2551.

4. - On secondary cohomology operation. II, Conference on Algebraic Topology, University of Illinois at Chicago Circle, pp. 117-133 (mimeographed).

5. A. L. Liulevicius, The factorization of cyclic reduced powers by secondary cohomology operations, Proc. Nat. Acad. Sci. U. S. A. 46 (1960), 978-981. MR 24 \#A2383.

6. W. S. Massey and F. P. Peterson, The cohomology structure of certain fibre spaces. I, Topology 4 (1965), 47-65. MR 32 \#6459.

7. - The mod 2 cohomology structure of certain fibre spaces, Mem. Amer. Math. Soc. No. 74 (1967). MR 37 \#2226.

8. R. J. Milgram, The structure over the Steenrod algebra of some 2-stage Postnikov systems, Quart. J. Math. Oxford Ser. (2) 20 (1969), 161-169.

9. - Steenrod squares and higher Massey products, Bol. Soc. Mat. Mexicana (2) 13 (1968), 32-57.

10. F. P. Peterson, $A$ note on $H$-spaces, Bol. Soc. Mat. Mexicana (2) 13 (1968), 30-31.

11. L. Smith, The cohomology of stable two stage Postnikov systems, Illinois J. Math. 11 (1967), 310-329. MR 34 \#8406.

12. N. E. Steenrod, Cohomology operations, Lectures by N. E. Steenrod and revised by D. B. A. Epstein, Ann. of Math. Studies, no. 50, Princeton Univ. Press, Princeton, N. J., 1962. MR 26 \#3056.

UNIVERSITY OF ROCHESTER, ROCHESTER, NEW YORK 14627 